\title{
Autoimmune Hepatitis: A Review with emphasis on its histomorphology
}

\author{
Pradhan SV ${ }^{1}$

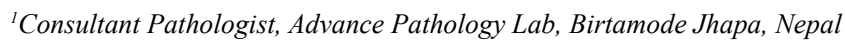

\author{
Keywords: \\ Antibodies; \\ Autoimmune Hepatitis; \\ Chronic liver disease
}

\begin{abstract}
Autoimmune hepatitis is a chronic inflammatory disorder characterized by periportal inflammation, hypergammaglobulinemia with elevated autoantibodies, and a dramatic response to immunosuppression. Various environmental and genetic influences can trigger the immune mediated destruction of the liver. A plethora of clinical presentations can be seen ranging from chronic indolent disease to fulminant hepatic failure. Autoimmune hepatitis does not have a pathognomonic feature, and its laboratory, serologic, and histologic manifestations are found in acute and chronic liver disease of diverse causes. Difficulties in distinguishing toxic, drug-related, virus-induced, and autoimmune causes of severe acute liver injury can result in misclassification.
\end{abstract}

Our paper discusses autoimmune hepatitis, giving a detailed overview of its clinical presentation, immunopathogenesis, emphasis on histomorphological changes and the diagnostic criteria.

\section{INTRODUCTION}

Autoimmune hepatitis (AIH) is an inflammatory disease of the liver characterized by circulating autoantibodies, hypergammaglobinemia, inflammation and necrosis of the liver. $^{1}$

The disease entity was first described by Leber in 1950 and has been given various terminologies since then. It is known as hypergammaglobulinemic chronic hepatitis active chronic hepatitis, chronic active hepatitis or autoimmune chronic active hepatitis and by other names such as chronic aggressive hepatitis, lupoid hepatitis and plasma cell hepatitis. The International Autoimmune Hepatitis Group designated "Autoimmune Hepatitis" as the most accurate and suitable term for the condition. ${ }^{2,3}$

\section{Correspondence:}

Dr. Seema V Pradhan, MD

Advance Pathology Lab, Birtamode, Jhapa, Nepal

Email:Pradhanseema30@yahoo.com

\section{Epidemiology}

Autoimmune Hepatitis is a relatively uncommon disease occurring in all races and in all geographical areas. According to different studies the prevalence of AIH in North America and western Europe is estimated to vary from 11-23\%.,3 The incidence of AIH among white in Northern Europe was $1.9 / 100000 .^{2}$ There are large groups of patients reported from South America, Alaska, Scandinavia and Austrailia. ${ }^{4}$ In Asia most report come from Japan with prevalence as high as 1:10000 cases. ${ }^{5} \mathrm{AIH}$ is now frequently reported in china ${ }^{6,7}$ few cases in Singpore ( 2 cases $) .{ }^{7}$ Rafeey et al reported $\mathrm{AIH}$ to be $5.6 \%$ of all childhood liver disease in Iran. ${ }^{8}$

Studies from India date back to 1998 where 10 cases were reported by Gohar et al. subsequently there are reports of AIH from different centers quoting the prevalence to be 1.5$6.4 \%$ of all liver disease..$^{9-11}$ 
There are no documented data regarding AIH in Nepal. But according to a report by $\mathrm{KC}$ et al, $12 \%$ of all chronic liver disease associated with hepatitis $\mathrm{E}$ is $\mathrm{AIH} .{ }^{12}$ The low prevalence of AIH in India and Nepal may be due to genetic or geographical makeup or an underestimation of prevalence of AIH as antibody studies are not accessible.

\section{Etiology and Pathogenesis}

\section{Infectious trigger/viruses}

The development of AIH is associated with viral Hepatitis A, Hepatitis B, Hepatitis C. AIH has developed after vaccination for Hepatitis $\mathrm{A}$ and $\mathrm{B}$. Other virus implicated to trigger the autoimmune process include human herpes virus, HIV, Measles virus, EBV, CMV. Molecular mimicry in $b$ cells level between a structural motif of CYP2D and HCV protein could explain the production of anti LKMI antibodies in $\mathrm{HCV}$ infected patients. ${ }^{13,14}$

\section{Drugs}

Nitrofurantoin, methylphenidate and atomoxetine, propylthiouracil, resperidone, rifampicin, pyrazinamide; betainterferon, doxycycline, minocycline, methyldopa. ${ }^{14,15}$ The AIH may be related to hepatotoxic effects of these chemiocal, upregulation of p450 immunoregulatory proteins or elated to drug acting as hapten modifying the hepatic protein and making them immunogenic. The drug induced AIH reverts after the drug is stopped. ${ }^{16,17}$

\section{Genetic Association}

AIH is a complex polygenic disease affected by various genetic and environmental triggers. HLA genes appear to play the dominant role in predisposing to AIH HLA DR3 is common in early onset, severe form of autoimmune disease, which often occurs in girls and young women. They are resistant to corticosteroid treatment and require liver transplantation. HLA DR4 is more common in adults and associated with increased incidence of extrahepaatic manifestation, milder disease and better response to corticosteroid treatment. ${ }^{1,2,18}$ Other HLA association are given in the Table $1 .{ }^{19}$

Twenty percent of AIH is associated with autoimmune polyendocrinopathy-candidiasis-ectodermal dystrophy syndrome (APECED) which follows Mendelian inheritance and prenatal genetic counseling is advised.

\section{Immunopathogenesis}

Liver is a part of lymphoid system with normal lymphocyte population residing in portal tracts. Various exogenous agents described above can upregulate expression of HLA I \& II in hepatocytes. The antigens activate the $T$ helper cells initiating the cascade of immune response leading to activation of cytotoxic $\mathrm{T}$ cells. The cytotoxic $\mathrm{T}$ lymphocytes recognizes the antigen presented by Class I molecules and also transform B lymphocytes to plasma cells. The autoantibodies release damages liver by release of interferon, complement or antibody dependent cytotoxic reaction (ADCC). CYP2D6 cytoplasmic enzyme is targeted by anti LKMI antibodies which damages the liver as shown in fig. $1 .{ }^{20-22}$

There is reduction in regulatory $\mathrm{T}$ cells responsible for dampening the immune response stopping the proliferation and effector function of autoreactive $\mathrm{T}$ cells. The $\mathrm{T}$ reg are defective in number and functions and their level is inversely proportional to the levels of anti SLA and anti LKM1 autoantibody titres.

\section{Classification}

The marked heterogeneity of $\mathrm{AIH}$ in regard to its variable presenting features, spectrum of disease severity, and presence of characteristic auto antibodies as well as response to therapy has led to several proposals for classification of the disease. The most approved classification includes type 1 and type 2 depending upon the antibodies present shown in Table 2. ${ }^{19}$

Type $1 \mathrm{AIH}$ constitutes $80 \%$ of total $\mathrm{AIH}$ and is characterized by the presence of antibodies to nuclei (ANA), smooth muscle (SMA) and Soluble Liver Antigen/Liver Pancreas antigen (SLA/LP). About $25 \%$ of these patient present with cirrhosis. These are associated with other autoimmune diseases like celiac disease, ulcerative colitis and autoimmune thyroid disease.

Type II AIH is characterized by presence of antibodies against a particular epitope on cytochrome P450 (IID6) enzyme located in liver and kidney microsomes (ALKM-1) and antibodies to liver cytosol antigen (ALC-1 or LC1). ${ }^{1,2}$ Most patients are children. Acute presentation can occur and progress to cirrhosis.

\section{Clinical manifestation}

The clinical presentation is heterogeneous. The spectrum of presentation range from no symptoms to debilitating symptoms and even fulminant hepatic failure. The patients are predominantly women $>70 \%$ of cases and $50 \%$ are younger than 40 years. The age at onset varies from infancy to elderly. Presenting feature may include, lethargy, fatigue, arthralgia , myalgia, anorexia, abdominal pain, nausea and dark urine. Children and elderly present with cirrhosis. ${ }^{1-3,18,19}$

Physical examination may be normal, but may reveal hepatomegaly, splenomegaly, jaundice, stigmata of liver disease. Rarely hirsutism, acne, obesity and amenorrheoa are seen. There is associated other autoimmune disorder like Hashimotos thyroiditis, type 1 DM, rheumatoid arthritis, 
Table 1: HLA Association in Autoimmune Hepatitis ${ }^{19}$

\begin{tabular}{|c|c|c|c|}
\hline HLA Association & Ethnicity & AIH type & No. of patient studied \\
\hline HLA- DRB180401 & $\begin{array}{l}\text { i. European and north American. } \\
\text { Increased susceptibility to type I } \\
\text { in Caucasian } \\
\text { ii. HLA DR } 3 \text { in younger ands } \\
\text { severe forms than HLA DR } 4\end{array}$ & I & 119 \\
\hline HLA DRB3*0101 & Europain and north America & I & 119 \\
\hline HLA DRB $1 * 0404$ & Mexican & I & 30 \\
\hline HLA DRB $1 * 0405$ & Japanese & - & 49 \\
\hline HLA DRB $1 * 07$ & Brazil & II & 28 \\
\hline HLA B14 & Germany & II & 19 \\
\hline
\end{tabular}

Table 2: Classificaton of autoimmune hepatitis ${ }^{19}$

\begin{tabular}{lll}
\hline Variable & Type I AIH & Type 2 AIH \\
& ANA & \\
Characteristic autoantibodies & SMA & Antibody against LKM \\
& AAA & Antibody against liver cytosol \\
& Antibody to SLA/LP antigen & \\
\hline Geographic variation & Worldwide & Worldwide, rare in North America \\
Age at presentation & Any age & Predominantly childhood and young adulthood \\
\hline Sex & Female in $75 \%$ of cases & Female in approximately $95 \%$ of cases \\
\hline Association with other autoimmune disorder & Common & Common \\
\hline Clinical severity & Broad range & Generally severe \\
\hline Histopathologic feature & Broad range & Generally advanced \\
\hline Treatment failure & Infrequent & Frequent \\
\hline Relapse after drug withdrawal & Variable & Common \\
\hline Need for long term maintenance & Variable & Approximately $100 \%$ \\
\hline
\end{tabular}

SLE, Ulcerative colitis and celiac disease in $20 \%$ of the patients.

\section{Laboratory Findings}

\section{Biochemical investigation}

There is marked increase in AST and ALT, but levels are generally $<500 \mathrm{u} / \mathrm{l}$ rarely reaching up to $1000 \mathrm{u} / \mathrm{L}$. Some patients may have elevated conjugated bilirubin and alkaline phosphtase necessitating workout for primary biliary cirrhosis (PBC), primary sclerosing cholangitis (PSC), viral hepatitis, biliary obstruction. Alkaline phosphatase rarely exceeds $4 \mathrm{x}$ the normal. $\mathrm{AIH}$ is associated with high hypergammaglobulinemia with selective increase in IgG 1.2-3.0 times. $^{1-3,18,19,23}$

\section{Serology}

Type $1 \mathrm{AIH}$ is characterized by the presence of antibodies to nuclei (ANA), smooth muscle (SMA) and Soluble Liver Antigen/Liver Pancreas antigen (SLA/LP). ${ }^{3}$ Type II
AIH is characterized by presence of antibodies against a particular epitope on cytochrome P450 enzyme located in liver and kidney microsomes and antibodies to liver cytosol antigen. ${ }^{1,19}$

\section{Seronegative AIH}

Approximately $10 \%$ to $15 \%$ of $\mathrm{AIH}$ is marker negative. ${ }^{23,24}$ Data suggest that seronegative AIHs similar to seropositive AIH with respect to demographics, aminotransferase levels at diagnosis or after treatment, response to therapy, and histologic parameters, including portal and lobular inflammation, interface activity, and centrilobular necrosis. ${ }^{25}$

\section{Liver Biopsy}

The diagnosis of AIH cannot be made without Liver biopsy in all cases whether acute or subclinical. Interface hepatitis is a pathologic hallmark of active AIH. It is especially prominent during disease flares. ${ }^{26-28}$ Interface hepatitis in AIH is characterized by a prominent lymphohistiocytic infiltrate at the portal tract mesenchymal-parenchymal 
Table 3: Simplified diagnostic criteria

\begin{tabular}{lcc}
\hline & Points \\
\hline & ANA or SMA or LKM $>$ & 1 \\
& $1: 40$ & \\
1. Autoantibodies & ANA or SMA or LKM $>$ & 2 \\
& $1: 80$ & \\
& SLA/LP Positive ( $>20$ units) & 2 \\
2. IgG & Upper normal limit & 1 \\
(or gamma-globulius) & $>1.10$ times normal limit & 2 \\
3. Liver histology & Compatible with AIH & 1 \\
4. Absence of viral & Typical for AIH & 2 \\
hepatitis & Yes & 2 \\
\hline
\end{tabular}

Table 4: Histologic component of the simplified criteria for the diagnosis of $\mathrm{AIH}^{37}$

\begin{tabular}{llc}
\hline Histology category & \multicolumn{1}{c}{ Description } & Points \\
\hline Typical & $\begin{array}{l}\text { Includes interface hepatitis, lym- } \\
\text { phocytic or lymphoplasmacytic } \\
\text { infiltrates in portal tracts extending } \\
\text { into the lobule, emperipolesis,a and } \\
\text { hepatocyte rosette formatio }\end{array}$ & 2 \\
Compatible & $\begin{array}{l}\text { Chronic hepatitis with lymphocytic } \\
\text { infiltration without } \\
\text { all the features considered typical }\end{array}$ & 1 \\
Atypical & $\begin{array}{l}\text { Atypical Includes evidence of } \\
\text { another diagnosis }\end{array}$ & 0 \\
\hline
\end{tabular}

junction with accompanying histologic evidence of liver cell damage. CD8-positive T cells are a dominant subset of lymphocytes within areas of interface hepatitis, and CD4positive $\mathrm{T}$ cells predominate within the portal tracts. ${ }^{18}$

Histologically, AIH has many faces depending on the course of the disease, the form of its initial presentation, its evolution, and effects of treatment. ${ }^{1,19,29}$

\section{ACUTE AUTOIMMUNE HEPATITIS}

Acute and fulminant forms of AIH were recognized by the IAIHG in 1992 when the diagnostic criteria waived the requirement for 6 months of disease activity to establish the diagnosis..$^{30}$ In acute $\mathrm{AIH}$, histology showed brisk, recent, ongoing immune-mediated hepatitis activity overlapping with evidence of chronicity including septal fibrosis and overt cirrhosis despite the lack of correlating clinical chronicity. ${ }^{30}$

The histology shows extensive interface hepatitis may be extensive with portal inflammation and diffuse lobular necroinflammation (fig. 2A) sparing the biliary tree. Hepatitis activity may show zone 3 accentuation (fig. 2B). Plasma cells typically predominate at the interface and throughout the lobules and portal areas, hence the name plasma cell hepatitis, eosinophils are also frequently seen. Evidence of hepatocellular injury and necrosis (ballooning degeneration, spotty hepatocyte necrosis, and apoptotic bodies) are common but not specific. Lobular disarray is present and bridging necrosis may occur. Injury may be followed by regeneration in the form of thickened hepatic plates and hepatic rosette formation. Most of these patients probably have a lobular "flare" in disease activity, which likely precipitated the clinical presentation as an acute hepatitis.

\section{FULMINANT AUTOIMMUNE HEPATITIS}

The features are more severe than acute AIH. There is bridging, zonal, and multilobular necrosis. Massive hepatocyte necrosis and drop out, parenchymal extinction, and stromal collapse may be present. ${ }^{31}$ Regenerative foci of hepatocytes may be present and mimicking cirrhosis.

\section{CHRONIC HEPATITIS}

AIH can assume a chronic hepatitis pattern of injury, with portal and periportal lymphoplasmacytic infiltrates and interface hepatitis. Plasma cells are often, but not always, prominent, and are sometimes seen singly and in clusters in the lobule. The severity of necroinflammatory activity is quite variable. Ballooning degeneration, spotty hepatocyte necrosis, and apoptotic bodies are common but not specific. ${ }^{29}$ Hepatocytes may form regenerating rosette-like structures.

\section{Pitfall in the diagnosis of AIH}

A. Zone 3 necrosis with or without portal inflammation

» not a specific finding of AIH

»Can also occur in viral and drug-induced hepatitis

B. Sparsity or absence of plasma cells in the inflammatory infiltrate

» Predominance of plasma cell infiltration is not specific for AIH

»Its presence supports the diagnosis and the finding is more common in this condition (66\%) than in chronic hepatitis B $(40 \%)$ or chronic hepatitis C (21\%). ${ }^{32,33}$

d does not occur in all patients with the disease (34\%), not present in seronegative cases

» The absence of plasma cells does not preclude the diagnosis. ${ }^{32}$

\section{Giant syncytial multinucleated hepatocytes}

» Giant syncytial multinucleated hepatocytes may be a dominant feature in AIH -"syncytial giant cell hepatitis";

»Also asociated with drug toxicity and viral infection, especially with the paramyxovirus.

D. Duct injury in AIHs

» Bile duct destruction is generally not prominent in $\mathrm{AIH}$,

» $12 \%$ of biopsies may show duct destruction.

» Lymphocytic infiltration of bile duct epithelium (see fig. 3) without duct loss can be seen in another $12 \% .34$

AUTOIMMUNE HEPATITIS AS PART OF AN OVERLAP SYNDROME 
$\mathrm{PBC}$ is a chronic cholestatic liver disease that is characterized by gradual destruction of the interlobular bile ducts that leads to damage of the hepatocytes. Approximately $10 \%$ of patients who have all the features of $\mathrm{PBC}$-positive antimitochondrial antibodies and cholestatic biochemical findings - present with additional features of AIH. These features include other autoantibodies such as smooth muscle antibodies, hypergammaglobulinemia with a fivefold elevation of AST or ALT, or a 10-fold elevation of the AST. Histologically, these patients may have lymphoplasmacytic interface hepatitis in addition to the typical florid duct lesions present in PBC. In addition to AIH-PBC overlap that is present at the time of diagnosis, a "sequential" overlap syndrome of AIH with PBC can occur. ${ }^{35}$

\section{AUTOIMMUNE HEPATITIS - POST TREATMENT}

In inactive, or subclinical AIH with raised transaminases biopsy show fibrosis, without or with mild lobular, portal, or interface necroinflammatory activity or a combination of these. Histologic improvement lags behind clinical and laboratory improvement by 3 to 6 months. In 55\% of cases, liver biopsy examination at the time of clinical and laboratory normality discloses residual interface hepatitis

The goals of corticosteroid therapy are to resolve symptoms, normalize laboratory tests, and improve histologic findings to normal, quiescent portal hepatitis, or inactive cirrhosis. Liver biopsy is the only means of confirming remission and it should be performed before drug withdrawal. ${ }^{34}$

Restoration of normal hepatic architecture during treatment is associated with a $20 \%$ frequency of relapse after drug withdrawal; the presence of portal hepatitis is associated with a $50 \%$ frequency of relapse; and the presence of interface hepatitis of any degree or progression to $20 \%$ frequency of relapse after drug withdrawal; the presence of portal hepatitis is associated with a 50\% frequency of relapse; and the presence of interface hepatitis of any degree or progression to cirrhosis is associated with an $87 \%$ to $100 \%$ frequency of relapse. ${ }^{17,32}$

\section{AUTOIMMUNE HEPATITIS - DIAGNOSTIC SCORING SYSTEMS}

The clinical spectrum of autoimmune hepatitis is very wide. Diagnosis is usually made by a combination of clinical, laboratory and histological features. Diagnostic scores can help both in the daily diagnostic work-up of patients, and in allowing comparability of clinical scientific studies. In 1992 the international autoimmune hepatitis group (AIHG) devised a diagnostic scoring system with the aim to categorize patients in groups of definite AIH and probable AIH. This was revised in 1999. This criteria allowed comparison of studies from different centers. Because these criteria are complex, insufficiently validated, and include a variety of parameters of questionable value, the IAIHG

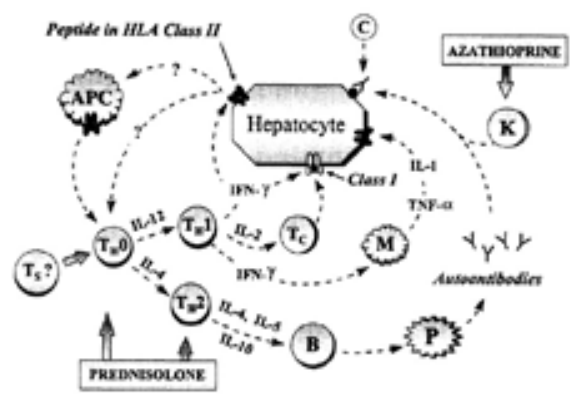

Figure 1: Pathogenesis of Autoimmune Hepattitis

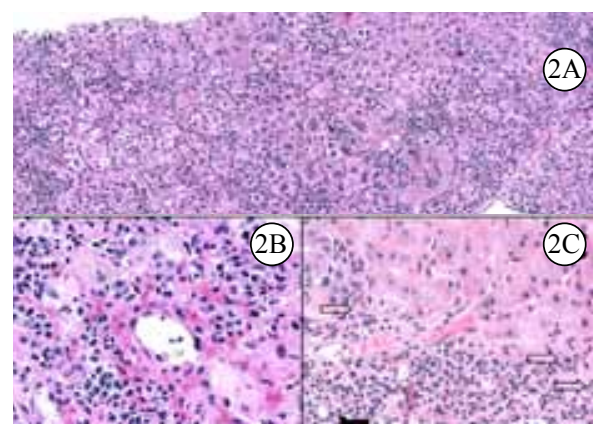

Figure 2: Autoimmune Hepatitis A. Diffuse parenchymal and portal inflammation (HE Stain, X50) B. Predominantly zone 3 involvement with plasma cell rich infiltrate and hepatocyte drop out and necrosis (HE Stain, X200) .C Interface activity(hollow arrows with minimal involvement of bile duct(HE Stain, X200).

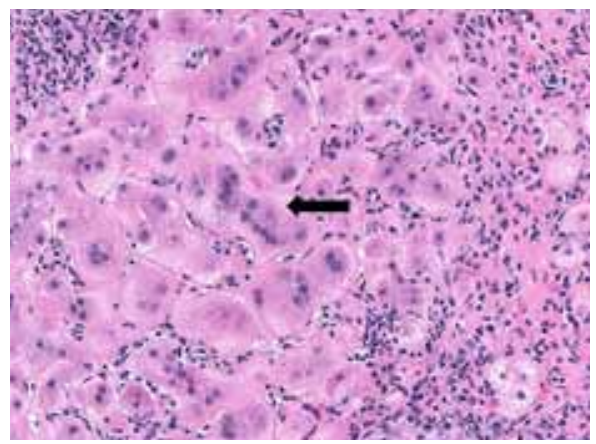

Figure 3: Liver parenchyma showing lymphoplasmacytic inflammation and several large multinucleated hepatocytes in the center of the field (HE Stain, X400).

decided to devise a simplified scoring system for wider applicability in routine clinical practice based on the data of patients with well-established diagnoses..$^{30,36,37}$

ANA, antinuclear antibody; SLA, soluble liver antigen; IgG, immunoglobulin G;

The new system condenses the liver histology criteria into three categories (Table 4). In this new system by Hennes and colleagues ${ }^{37}$, the presence of histologic evidence of hepatitis is a necessary finding to categorize patients as having either "definite" or "probable" AIH in an objective manner. A positive weighting was given to female gender, 
hepatocellular rather than cholestatic damage, and the presence of autoantibodies. The diagnosis of definite AIH required a liver biopsy.

However, all diagnostic scores have limitations in individual cases .The simplified score cannot detect for example; patients with coexistence of AIH and HCV. The latter could be a problem for areas with high endemicity of $\mathrm{HCV}$ infections. Twenty to forty percent of patients with chronic $\mathrm{HBV}$ of $\mathrm{HCV}$ are persistently positive for various autoantibodies, usually at low titers (B1:20 or 1:40). ${ }^{3}$ Conversely, patients with AIH sometimes have a false-positive test for anti-HCV antibodies but will have undetectable HCV-RNA. Three categories of patients with potential concurrent $\mathrm{AIH} /$ hepatitis $\mathrm{C}$ may be identified:

» patients with true $\mathrm{AIH}$ and false-positive anti- $\mathrm{HCV}$ antibodies (undetectable HCV-RNA);

» patients with true $\mathrm{HCV}$ and autoantibodies at low titers, but no other signs of AIH;

» patients with true $\mathrm{HCV}$ and features of AIH including young age, female gender, high

» autoantibody titers (41:320), hypergammaglobulinemia, and history of extrahepatic autoimmune disorders.

Distinction of chronic viral hepatitis from AIH is important, because interferon therapy can exacerbate autoimmune conditions, and corticosteroids can enhance viral replication. In a study of the IAIHG, the simplified score was found $97 \%$ specific and $88 \%$ sensitive. ${ }^{37}$

The revised scoring system might be useful in the evaluation of "difficult" cases with few or atypical features of AIH after exclusion of other diagnoses, while the simplified scoring system - simpler in its determination - can be used in aetiologically distinctive cases with concurrent immune trappings in order to exclude AIH..The various studies strongly shows that as there is no single test for $\mathrm{AIH}$, histology is very important where there is any doubt about the diagnosis, and in patients where there may appear to be more than one disease, histology is the best guide of treatment. ${ }^{30,36-38}$

\section{CONCLUSION}

Early diagnosis and treatment of AIH shows a dramatic recovery of the paatients. But the diagnosis of $\mathrm{AIH}$ is challenginsg because clinical picture is heterogenous and there is no specific test applicable for all patients. The liver biopsy should be mandatory in patients with liver disorders in whom there is evidence of autoimmunity and absence of evidence of HBV or HCV markers. The cooperation between clinicians and pathologists seems mandatory.

\section{REFERENCES}

1. Makol A, Watt KD. Autoimmune hepatitis: A review of current diagnosis and treatment. Hepatitis Research and Treatment 2011; 3901916.Epub
2. Manns MP, Vogel A. Autoimmune hepatitis, from mechanisms to therapy. Hepatology 2006;43:S132-44.

3. McFarlane IG. Definition and classification of autoimmune hepatitis. Semin Liver Dis 2002;22:317-24.

4. Ansgar W.L, Giorgina MV, Lohse AW, Mieli-Vergani G, Chowdhary VR.. Autoimmune hepatitis Journal of Hepatology 2011;55:171-82.

5. Toda G, Zeniya M, Watanabe F et al. Present status of autoimmune hepatitis in Japan-correlating the characteristics with international criteria in an area with a high rate of HCV infection. Japanese National Study Group of Autoimmune Hepatitis . J Hepatol 1997;26:1207-12.

6. Lam KC, Lai CL, Wu PC, Todd D. Etiological spectrum of liver cirrhosis in the Chinese. J Chronic Dis 1980;33:375-81.

7. Basir N, Yew TG, Telisinghe PU et al. Autoimmune hepatitis in children: a report of two cases; Singapore Med J 2011;52:e27-30.

8. Rafeey M, Kianrad M, Hasani A; Autoimmune hepatitis in Iranian children: Indian J Gastroenterol 2007;26:11-3.

9. Choudhuri G, Somani SK, Chalamalasetty SB, Baba CS, Alexander G. Autoimmune hepatitis in India: profile of an uncommon disease. BMC Gastroenterol 2005;5:27

10. Gupta R, Agarwal SR, Jain M, Malhotra V, Sarin SK. Autoimmune hepatitis in the Indian subcontinent: 7 years experience; J Gastroenterol Hepatol. 2001;16:1144-8.

11. Gohar S, Desai D, Joshi A et al; Autoimmune hepatitis: a study of 50 patients.Indian J Gastroenterol. 2003;22:140-2.

12. KC S, Sharma D, Basnet BK, Mishra AK. Effect of Acute Hepatitis E Infection in Patients with Liver Cirrhosis. JNMA 2009;48:226-9.

13. Skoog SM, Rivard RE, Batts KP, Smith CI. Autoimmune hepatitis preceded by acute hepatitis A infection. Am J Gastroenterol 2002;97:1568-9

14. Lewis JL, Zimmerman HJ. Drug-induced autoimmune liver disease. In: Krawitt EL, Wiesner RH, Nishioka M, eds. Autoimmune liver diseases. 2nd ed. Elsevier: Amsterdam;1998: pp627-49.

15. Gough A, Chapman S, Wagstaff K,et al. Minocycline induced autoimmune hepatitis and systemic lupus erythematosus-like syndrome. BMJ 1996;312:169-72.

16. Czaja AJ. Current concepts in autoimmune hepatitis. Ann Hepatol 2005;4:6-24.

17. Pelli N, Setti M. Atorvastatin as a trigger of autoimmune hepatitis. J Hepatol 2004;40:716.

18. Malik T.A, Saeed S. Autoimmune Hepatitis: A Review. J Pak Med Assoc 2010;60:381-7.

19. Krawitt EL . Autoimmune Hepatitis; Review article. N Engl J Med 2006;354:54-66.

20. Francesco BB, Paolo M, Luigi M. New autoantibodies and autoantigens in autoimmune hepatitis. Clin Liver Dis 2002;6:785-97.

21. Mc Farlane IG. Pathogenesis of Autoimmune hepatitis.Biomed \&Pharmacother 1999;53;255-63.

22. Manns MP, Czaja AJ, Gorham JD et al. Diagnosis and management of autoimmune hepatitis. Hepatology, 2010; 51:2193-213.

23. Czaja AJ, Carpenter HA, Santrach PJ et al. The nature and prognosis of severe cryptogenic chronic active hepatitis. Gastroenterology 1993;104:1755-61.

24. Czaja AJ. The variant forms of autoimmune hepatitis. Ann Intern Med 1996;125:588-98.

25. Mehendiratta V, Mitroo P, Bombonati A et al. Serologic markers do not predict histologic severity or response to treatment in patients with autoimmune hepatitis.Clin Gastroenterol Hepatol 2009;7:98103.

26. Batts KP, Ludwig J. Histopathology of autoimmune hepatitis, primary 
biliary cirrhosis, and primary sclerosing cholangitis. In: Krawitt EL, Wiesner RH, Nishioka M, eds. Autoimmune liver diseases. 2nd ed. Elsevier: Amsterdam;1998. pp115-40.

27. Da Dienes HP. Viral and autoimmune hepatitis. Morphologic and pathogenetic aspects of cell damage in hepatitis with potential chronicity. Veroff Pathol 1989;132:1-107.

28. Washington KM. Autoimmune liver disease: overlap and outliers. Mod Pathol 2007;20:S15-30.

29. Guindi M, Histology of Autoimmune Hepatitis and its Variants; Clin Liver Dis 2010;14:577-90

30. Burgart LJ, Batts KP, Ludwig J, Nikias GA, Czaja AJ. Recent onset autoimmune hepatitis: biopsy findings and clinical correlations. Am J Surg Pathol 1995;19:699-708.

31. Czaja AJ, Carpenter HA. Autoimmune hepatitis. In: Macsween's pathology of the liver. 5th edn. Churchill Livingstone: Edinburgh;2007.pp493-515.

32. Czaja AJ, Carpenter HA. Sensitivity, specificity and predictability of biopsy interpretations in chronic hepatitis. Gastroenterology 1993;105:1824-32.

33. Carpenter HA, Czaja AJ. The role of histologic evaluation in the diagnosis and management of autoimmune hepatitis and its variants. Clin Liver Dis 2002;6:397-417.

34. Czaja AJ, Carpenter HA. Autoimmune hepatitis with incidental histologic features of bile duct injury. Hepatology 2001;34:659-65.

35. Johnson PJ, McFarlane IG. Meeting report. International Autoimmune Hepatitis Group. Hepatology 1993;18:998-1005.

36. Alvarez F, Berg PA, Bianchi FB et al. International Autoimmune Hepatitis Group Report: review of criteria for diagnosis of autoimmune hepatitis. J Hepatol 1999;31:929-38.

37. Hennes EM, Zeniya M, Czaja AJ et al.Simplified criteria for diagnosis of autoimmune hepatitis. Hepatol 2008;48:169-76

38. Gatselis NK, Zachou K, Papamichalis $\mathrm{P}$ et al. Comparison of simplified score with the revised original score for the diagnosis of autoimmune hepatitis: A new or a complementary diagnostic score. Dig Liver Dis 2010; 42:807-12. 